\section{A modification to a program for the analysis of intragroup interaction to allow inter- facing with the SPSS package}

\author{
W. P. DODD \\ Computer Center, \\ University of Birmingham, Birmingham, England \\ and \\ E. JONES \\ Subdepartment of Ethology, \\ University of Birmingham. Birmingham. England
}

In an earlier paper (Dodd \& Jones, 1974), we described a computer program for the analysis of a set of positional data for a colony of macques. The data set consisted of the position of each subject at regular time intervals over an extended period. We obtained many data sets and, having analyzed each set using the above program, we wished to study the progress of the colony from period to period. This required a statistical analysis of many of the quantities obtained. Instead of writing our own special-purpose programs, we used the SPSS package (Nie, Bent, \& Hull, 1970). In this note we outline the modifications made to the earlier program so that it produces a file suitable for input to the SPSS package.

An SPSS input file is in two distinct sections, a "data-definition" section and a "case data" section. The data-definition section contains a list of the variables in the case data section, their input format, and a set of labels to be associated with each variable name and with the values of certain variables. This information, with one exception, is either constant for the present application or can be easily constructed; and a new subroutine, HEADER, optionally called from subroutine CHIMP, has been added to write it out. The exception is the \# of CASES card, which is produced with a blank data field, to be filled in prior to the SPSS run. A listing of the data-definition section for a typical application of the program is shown in Figure 1 . In the case data section, the user either produces one large record for each observation period or subdivides this into separate records for each subject. We chose the latter course since this simplified the subject-dependent tests in SPSS. However, we also produced an "average" record for each observation period, since SPSS does not have a facility for combining separate records. A further consequence of the decision to produce separate records is that each record has to include some variables de-

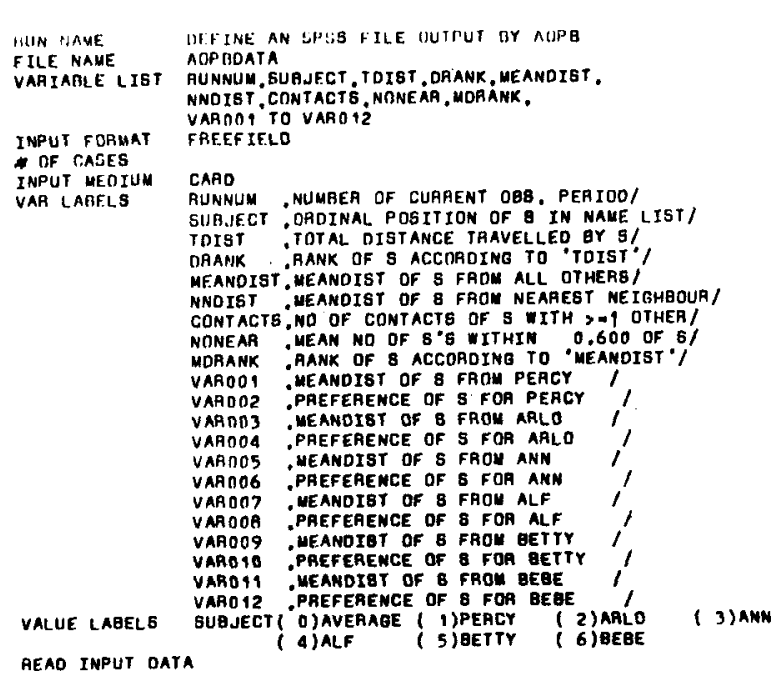

Figure 1.

pendent on more than the current subject, for example, ranking according to distance traveled. A list of variables in a typical case record is given on the VARIABLE LIST card in Figure 1, with the expanded title of each variable given on the VAR LABELS cards. The list of variables includes some quantities which were not calculated in the earlier version of the program: in particular, the "preference" ranking for the current subject of each of the other subjects and a number of measures of the sociability of the subject. The preference ranking is calculated from the mean inter-subject distances, the nearest subject having a preference rank of 1 . The other variables are self-explanatory, with the possible exception of NONEAR, which is the mean number of subjects within a user-supplied distance (in this example $.60 \mathrm{~m}$ ) of the subject. All these additional variables are also written to the standard output channel.

A listing of the program, including full details of $\mathrm{I} / \mathrm{O}$ formats and all internal variables, may be obtained without charge from: W.P. Dodd, Computer Center, Birmingham University, Birmingham, B15 2TT, United Kingdom.

\section{REFERENCES}

DodD, W. P., \& Jones, E. A program for the analysis of intragroup interaction based on spatial dynamics. Behavior Research Methods \& Instrumentation, 1974, 6, 497-503.

NiE, N., BENT, D. H., \& HulL, C. H. SPSS: Statistical package for the social sciences. New York: McGraw-Hill, 1970. 